\title{
Analysing Mobile Random Early Detection for Congestion Control in Mobile Ad-hoc Network
}

\author{
Saurabh Sharma ${ }^{1}$, Dipti Jindal ${ }^{2}$, Rashi Agarwal $^{3}$ \\ ${ }^{1,3}$ Deparment of Computer Science Engineering, Sharda University, India \\ ${ }^{2}$ Deparment of Computer Science Engineering, Sky line College, Noida, India
}

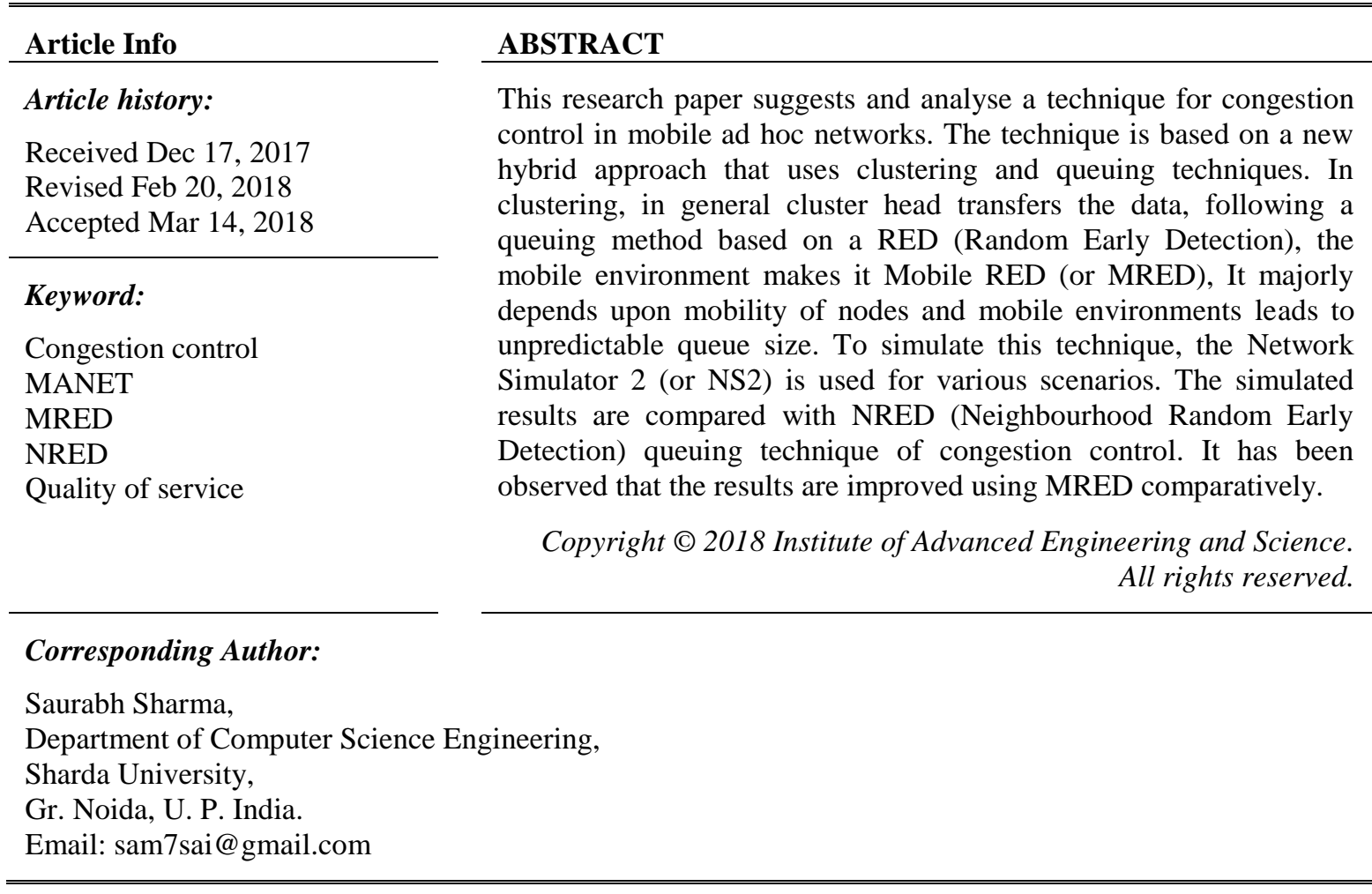

\section{INTRODUCTION}

An ad hoc network is a collection of wireless devices(referred to as nodes) which communicate with each other using shared wireless medium without intermediate infrastructure. When nodes move and change their locations in these specialized networks then this category is called Mobile Ad hoc Network or MANET. In general, mobile nodes organize themselves to form a network over radio links while communicating with each other. One of the advantages of this network is that it can be easily deployed anywhere as it is defined in [1], [2], but a large amount of real-time traffic requires high bandwidth. The reason is that these nodes share a priori protocol information along with up-gradation of information in terms of parameters which takes part in communication, for example the evolving \& scaling or expanding parameter list causes congestion [3]. Congestion not only affects the integrity of data but also decreases overall bandwidth and throughput of the links \& ultimately reduces QoS of network. To avoid congestion, a lots of work has been done earlier, such as Cluster Based QoS (Quality of Service) Routing [4], Cross Layer congestion control [5] \& Queuing Technique e.g., Neighborhood Random Early Detection (NRED) [6] as shown in Table 1. The main objective of all these efforts is to limit the delay and buffer overflow caused by congestion, and improve the communication performance, the enhanced form of Queing technique will be used in this research paper in which nodes are in the mobile trajectory and NRED changed in to MRED, Diffrence in the approach of droping packet when it goes up from the threshold value. It not only reduces congestion but also improves QoS. A description on Background, Problem and the proposed solution. 


\subsection{Background}

A number of researches have been done to give a solution for congestion control. In Table-1 there is a list of congestion control techniques, different columns represents author, publication year, research topic, methodology used and problems noted in applying the methodology. In Table-1, problems related with TCP fast start, RED, ARED revised ARED, improved ARED, weighted RED, Cross layer design approach, NRED, and CBQR (cluster based QoS routing protocol) are tabulated. In TCP fast start approach [7], a sender temporarily stores congestion information to start the new connection from a larger initial window size. The advantage is that, it avoids the slow start penalty for each page download. However, with this methodology, there is a risk of performance degradation when the cached information is stale (or old). And, the new route is used for next transaction. The Active Queue Management (AQM) technique, named RED in [8], reduces packet loss, attains high throughput and prevents global synchronization as discussed in Table 1. The RED gateway drops the packets when the average queue size is greater than maximum threshold value Feng et al. [9] presents the original ARED. A revised version of ARED (Adaptive RED) is presented by Floyd et al. [10], which is also named as Adaptive RED. An improved ARED technique described in [6] optimizes the bounds on the maximum drop probability and adjusts the lower threshold of the exponential averaging weight on linear stability conditions. The techniques, mentioned above, have been applied successfully in wired networks, to improve the TCP performance in ad hoc networks. Several techniques have been proposed emphasizing on addressing link breakages, routing algorithm failures and mobility [11].

\subsection{The problem}

The congestion control problem in ad-hoc wireless networks, described in Antonopoulo et al [12] identifies that the main cause for performance degradation in wireless network is excessive congestion. For such networks the utilization of the cross-layer design approach is advocated. They also argued that the layered approach of the OSI/ISO model is not sufficient enough to provide substantial performance enhancement in wireless networks with dynamic nature. To provide a promising solution $\mathrm{Xu} \mathrm{K}$. et al [11] proposed NRED (Neighborhood RED) technique, which is an extension of original RED [8] developed for wired networks. An NRED brings the concept of distributed neighborhood queue. It is given in the table given herewith degree algorithm, WCA (Weighted Clustering Algorithm) [13], [14] etc.Thus, Congestion control is the main problem area and addingly queuing too, which has not been worked together for MANETS earlier, which raises a requirement of a technique for MANETS .

\subsection{The proposed solution}

To overcome the problem a hybrid technique is introduced in this research. We refer to this technique as Mobile RED (Mobile Random Early Detection) and abbreviated as MRED. In this technique the original ARED (Adaptive RED), [10] is applied instead of RED [8] at the cluster head nodes in a clustered network. The difference in, RED, ARED, NRED \& MRED, is the way their drop probabilities. The RED gateway starts dropping the packets when the average queue size reaches the maximum threshold value while in ARED (Adaptive RED), which dynamically changes the range of maximum drop probability $\mathrm{P}_{\max }$ according to different network scenarios and adjusts $P_{\max }$ to limit average queue size $Q_{\text {ave }}$ in a steady range, thus, it is more suitable for ad hoc networks (dynamic topology) in the proposed method. The scenario is supposed to be simulated on MANET type of networks in which not only cluster nodes but also cluster head regularly change their location. MRED also works same as ARED [15], [16]. But, the ever changing position of Cluster head changes the values which is the main challenge, its delt in this research. As far as current research in concerned. In Abinasha Mohan et. al. [17] a noval work on queue management was done using basic RED technique. They have given a joined early congestion based solution on cross layer designed to optimize congestion control. S. Subharmanam in [18] has suggested predictive congestion control using a predictive congestion index of a node as ratio of current queue occupancy over the available total queue size $\&$ that node. It completed using AODV protocol and proactively defined \& finds congestion. Several research works are going on, in the currecnt scenario keeping power and energy as main parameters .Work done in [19] is done using integration in optical \& wireless networks which also proposes a power consumption model for such type of networks [20].

The paper is consisting of 6 sections in which Section 1 contains introduction about the problem Section 2 gives the Research method which gives more clarity about the relevance of the problem with several schemes, modules and techniques which are evolved earlier to remove congestion to give better QoS (Quality of Service). Section 2 also gives clear idea about the protocols discovered in current scenario for the congestion control by giving simultaneous explanation of RED, ARED, NRED \& MRED. Section 3 gives the result analysis for efficient clustering technique and the proposed clustering and queuing combination, making it as a hybrid technique MRED. All experiments, scenario simulation and its analysis done explained in Section 3 and 4 simultaneously. Section 5 gives conclusion followed by the references. 


\section{RESEARCH METHOD}

\subsection{Mobile random early detection (OR MRED)}

The congestion in an ad hoc network can be traced to the entire space around the node because in ad hoc network node has to compete for the channel requirements with the nodes that lie in the same. "Neighbourhood" is the name given for this "space" in [11] to name it as NRED. NRED is compared with MRED in this research, in which MRED technique is applied on the cluster head in the clustered network. Cluster-head contains the information of its member node as well as of other cluster-heads, is the reason why we apply MRED on cluster-head. It will also reduce the load from the member nodes in a cluster by calculating the average queue size or we can say channel utilization. The queue size on the cluster-head nodes determines the degree of congestion in network. For this first we have to choose the cluster-head first, as shown in Figure 1 [2].

The objects in one cluster are similar in terms of synchronisation than the objects that lies in other cluster. Every cluster selects a cluster head and all the other nodes which lie in the transmission range of that cluster-head are called the member nodes of that cluster, as shown in Figure 1 [1]. Several algorithms are proposed for the selection of cluster-head, but we are using the highest degree algorithm to find the clusterhead.

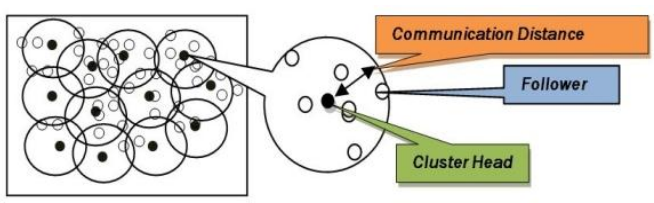

Figure 1. Selecting cluster head

\subsection{Cluster-head congestion detection}

After the cluster-head selection these cluster-heads have to detect the congestion in the network. It is similar to the congestion detection in NRED except that the congestion is detected at the cluster gateway or cluster head nodes. A brief overview is provided here for congestion detection in ad hoc network. As it is difficult to get the actual queue size of node in ad hoc network due to change in traffic pattern and network topology, so, channel utilisation is used to measure the queue size in ad hoc network and there is also a direct relationship between channel utilization and input- output queue size and there are 5 different radio states that are monitored by the nodes. These radio states are: a) Transmit, b) Receive, c) Carrier sensing busy, d) Virtual carrier sensing busy (e.g. deferral to RTS, CTS etc.), and e) Idle. Figure 2 show the flow diagram for Cluster head selection.

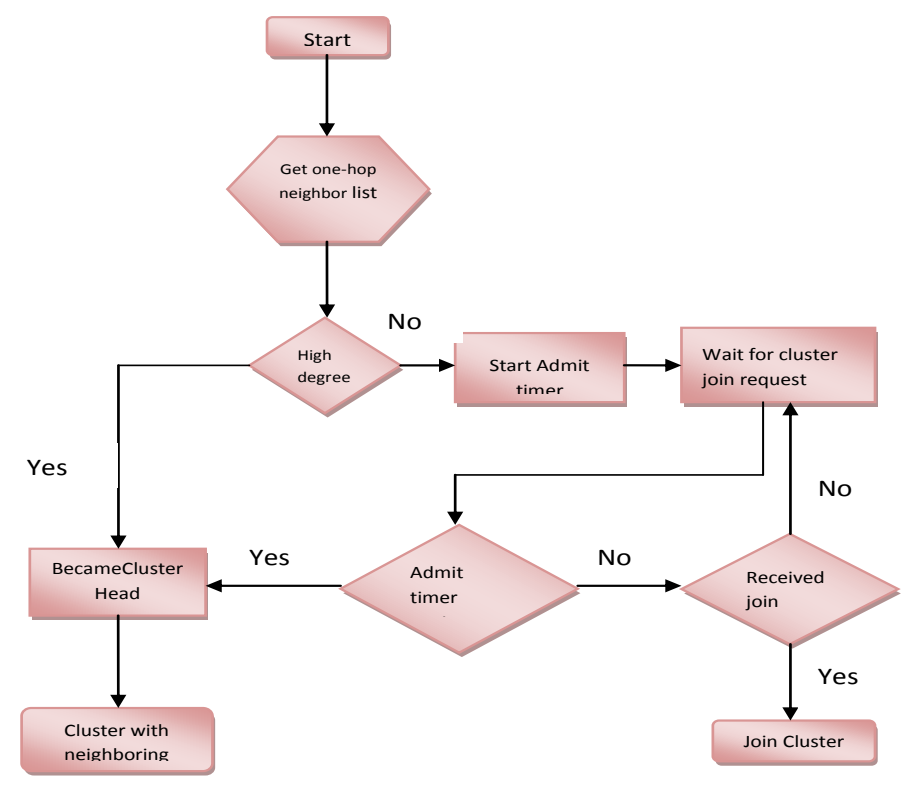

Figure 2. Flow diagram for cluster head selection 
These radio states are divided into 3 categories in, where states 1) and 2) contribute of node to the total channel utilization by the nodes. States 3) and 4) are the contribution of the node's neighbors to the channel utilization, and state 5) is assumed as empty queue. In original NRED, a node estimates 3 channel utilization ratios, i.e. total channel utilization ratio $\left(\mathrm{U}_{\text {busy }}\right)$, transmitting ratio $\left(\mathrm{U}_{\mathrm{tx}}\right)$ and receiving ratio $\left(\mathrm{U}_{\mathrm{rx}}\right)$ and maintains the logs for time period in each 5 radio states as. $T_{t x}, T_{r x}, T_{c s}, T_{v c s}$ and $T_{i d l e}$. Let $T_{\text {int }}$ represents the total time period spent at each state. Then utilization ratios become:

$$
\begin{aligned}
& U_{\text {busy }}=\frac{T_{\text {int }}-T_{\text {idle }}}{T_{\text {int }}} \\
& U_{t x}=\frac{T_{t x}}{T_{\text {int }}} \\
& U_{r x}=\frac{T_{r x}}{T_{\text {int }}}
\end{aligned}
$$

Where,

$$
\begin{aligned}
& \mathrm{T}_{\mathrm{int}}=\mathrm{T}_{\mathrm{tx}}+\mathrm{T}_{\mathrm{rx}}+\mathrm{T}_{\mathrm{cs}}+\mathrm{T}_{\mathrm{vcs}}+\mathrm{T}_{\mathrm{idle}} \\
& \mathrm{U}_{\text {busy }}=\text { cluster-head queue size. } \\
& \mathrm{U}_{\mathrm{tx}}=\text { outgoing queue channel bandwidth usage, and } \\
& \mathrm{U}_{\mathrm{rx}}=\text { incoming queue channel bandwidth usage }
\end{aligned}
$$

The network is said to be in early congestion state if $U_{\text {busy }}$ exceeds its threshold value. Now this channel utilization is translated into an index of the queue size by using

$$
\mathrm{q}=\frac{\mathrm{U}_{\text {busy }} * \mathrm{~W}}{\mathrm{C}}
$$

Where,

$\mathrm{W}$ is channel bandwidth in bps

$\mathrm{C}$ is average packet size in bits (Constant)

The variable $\mathrm{q}$ is not dimensionally correct, and it is expressed in pkts/sec rather than packets. It is only a scaling factor that affects the choice of the values for minimum and maximum threshold $\left(\mathrm{Th}_{\min }\right.$ and $\left.\mathrm{Th}_{\max }\right)$.

Similarly, $\mathrm{q}_{\mathrm{tx}}$ and $\mathrm{q}_{\mathrm{rx}}$ can be calculated using $\mathrm{U}_{\mathrm{tx}}$ and $\mathrm{U}_{\mathrm{rx}}$.Now, the average queue size is

$$
\operatorname{avg}=(1-w q) * a v g+w q * q
$$

Initially avg is 0 and $\mathrm{w}_{\mathrm{q}}$ is weight parameter. Similarly, we can also get avg $\mathrm{t}_{\mathrm{tx}}$ and avg $\mathrm{g}_{\mathrm{rx}} \mathrm{using}_{\mathrm{tx}}$ and $\mathrm{q}_{\mathrm{rx}}$. $\operatorname{avg}_{\mathrm{tx}}$ and $\operatorname{avg}_{\mathrm{rx}}$ are the average queue size of the incoming and outgoing queue.

\subsection{Cluster-head congestion notification}

Under MRED, the cluster gateway or Cluster head node checks the estimated average queue size avg periodically and compares it with a minimum threshold $\mathrm{Th}_{\min }$. If queue is larger than threshold, early congestion is detected. Then the node calculates a drop probability $\mathrm{p}_{\mathrm{b}}$ based on the average queue size and broadcasts it to other cluster-head. This paper also replaces the specified target range of average queue size as

$$
q_{\text {target }}=\left[T h_{\min }+0.4\left(T h_{\max }-T h_{\min }\right), T h_{\min }+0.6\left(T h_{\max }-T h_{\min }\right)\right]
$$

The bound on $\mathrm{q}_{\text {target }}$ and $\mathrm{p}_{\max }$ is based on ARED [10]. Here, we present the algorithm for calculating $\mathrm{p}_{\mathrm{b}}$ using pseudocode.

\footnotetext{
Algorithm 1: Calculating Drop Probability $p_{b}$

Saved Variables:

avg: average queue size

Fixed Parameters:
} 


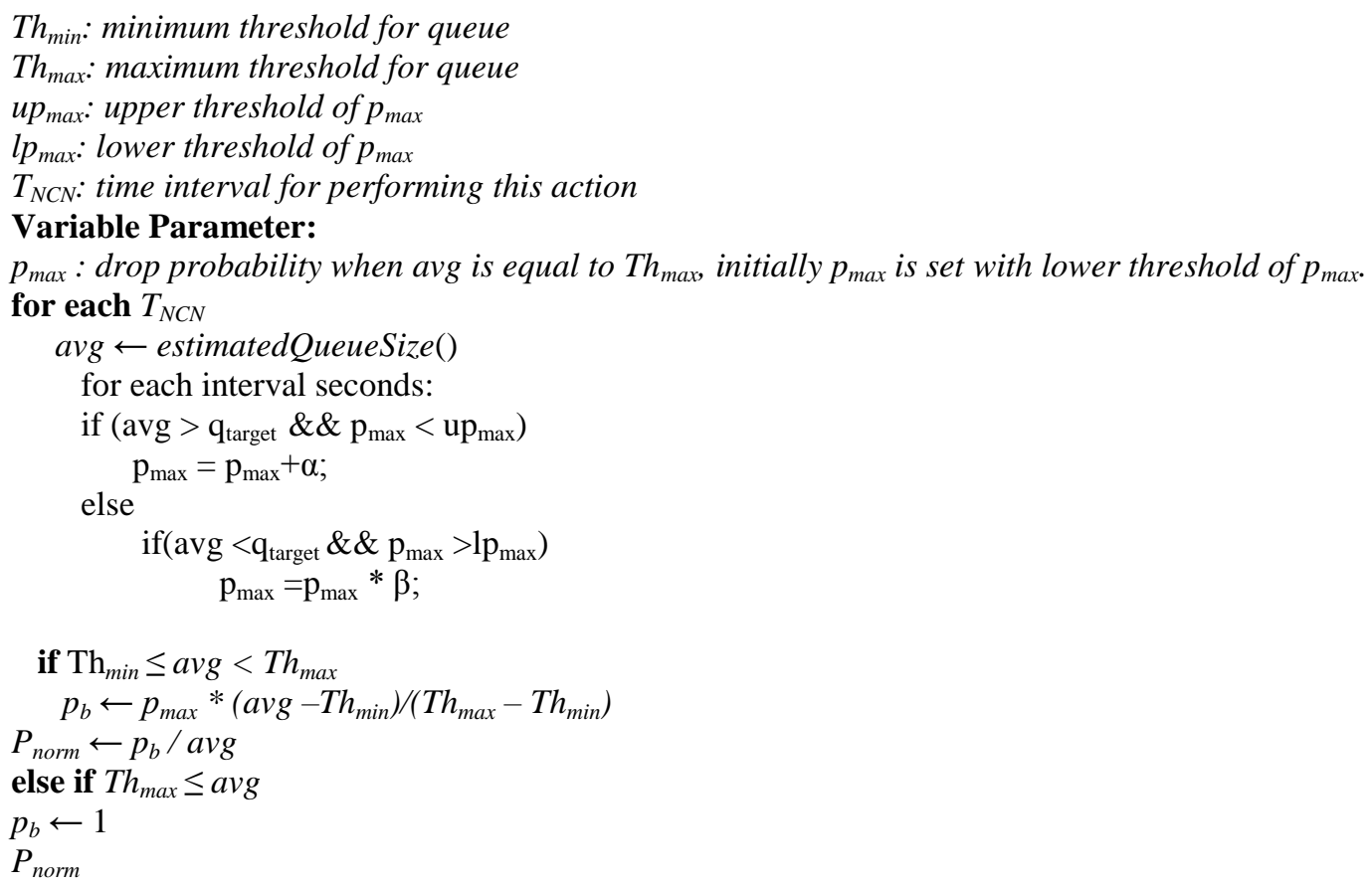

Where, $P_{\text {norm }}$ is normalized probability and the value used by estimated Queue Size() is calculated from channel utilization as index queue size.Three fields, packet Type, $\mathrm{P}_{\text {norm }}$, and lifetime, are used by the NCN packets as in [11]. The field "packet Type" represents a NCN packet. Cluster-heads calculate their local drop probability by using Normalized Probability i.e. $\mathrm{P}_{\text {norm }}$ and packet dropping is stopped after lifetime period. In case of multiple NCN packets are received largest $P_{\text {norm }}$ is stored at $P_{\text {norm }}$ field.

\subsection{Cluster gateway/head packet drop}

Since congestion is detected and notified to other cluster-heads, now, we explain how these clusterhead nodes cooperatively drop packets to realized the expected drop probability $\mathrm{p}_{\mathrm{b}}$ over the distributed queue. Overall drop probability local share of cluster-heads is calculated and is proportional to its queue size. In our clustered model, there are two queues that are associated at each cluster-head node, i.e. the outgoing queue and incoming queue. Both the queues calculate and implement packet drop probability separately. For this we are using the same pseudocode as used in [8].

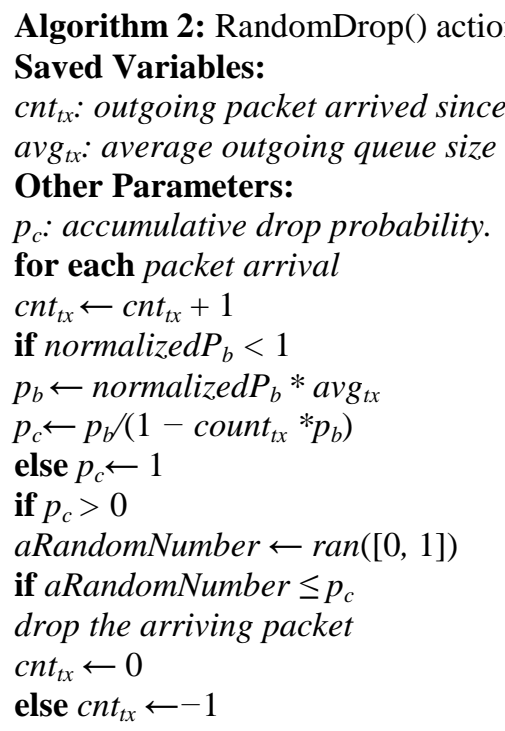

Random number between 0 and 1 are generated by using the function ran $([0,1])$ in the above pseudocode. Same action is performed on incoming queue by using avg $\mathrm{r}_{\mathrm{rx}}$ and $\mathrm{cnt}_{\mathrm{rx}}$ in place of avg $\mathrm{g}_{\mathrm{tx}}$ and $\mathrm{cnt}_{\mathrm{tx}}$. So, the parameters of mobility is also checked and used as the parameters to check droping of packets. 
The reason why we apply MRED on cluster- head nodes are:

a. Cluster-head contains the information of its member node as well as of other cluster-heads.

b. It will reduce the bourdon from the member nodes, in a cluster, of calculating the average queue size or we can say channel utilization. The queue size on the cluster-head nodes determines the degree of congestion in network

\section{EXPERIMENTAL SET UP AND RESULTS ANALYSIS}

\subsection{Scenario}

The scenario of this model consists of very small experimental setup of 17 mobile nodes, 2 gateways are tested here on the Network Simulator-2(NS2). The topology is a rectangular area with $1000 \mathrm{~m}$ length and $1000 \mathrm{~m}$ width. The two gateways are placed on each side of the area; their $\mathrm{x}, \mathrm{y}$-coordinates in grid are $(150,280),(800,250)$. All simulations are run for 150 seconds of simulated time. Four of the 17 mobile nodes are constant bit rate traffic sources as shown in the table in Figure 3. They are distributed randomly within the mobile ad hoc network. After this time the sources continue sending data until one second before the end of the simulation.

\begin{tabular}{ll}
\hline PARAMETER & VALUES \\
\hline Simulation time & $150 \mathrm{sec}$ \\
Topology size & $1000 \mathrm{X} 1000$ \\
No. of nodes & 17 \\
No. of clusters & 2 \\
Node mobility & 0 to $20 \mathrm{~m} / \mathrm{sec}$ \\
Routing Protocol & DSDV \\
Frequency & $11 \mathrm{MHz}$ \\
Traffic type & CBR \\
MAC & IEEE 802.11 \\
Mobility model & Random Waypoint \\
Max. no. of packets & 10000 \\
Pause time & $10 \mathrm{sec}$ \\
\hline
\end{tabular}

Figure 3. Parameters used

\subsection{Cluster formation $\&$ cluster changes}

The nodes in the cluster are mobile in nature thus the cluster nodes as well as the heads changes their positions unknowingly. The cluster change variation and cluster head change variation with respect to node mobility are shown in fig 4 . We can observe that the network is more stable in lower mobility scenarios. The simulations for these results were carried with No. of node $=17$ and Topology size $=1000 \mathrm{x} 1000$. As the node mobility increase the cluster change increase pause time for mobile nodes the changes also decrease, thus, cluster head changes and cluster head changes also decreases if node mobility decreases and pause time increases from 1 to 100 secs which can be easily seen in the data set kept in Table 2 [2], graph shown in Figure 4 a drop in pause time as per dereased mobility.

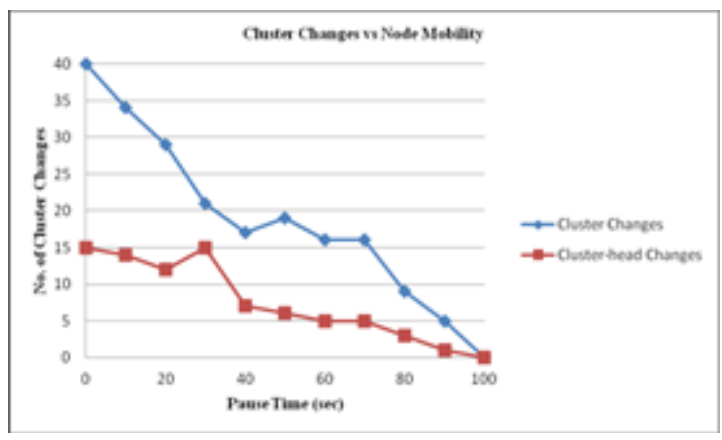

Figure 4. Cluster changes vs node mobility 
Table 2. Cluster Changes Vs Node Mobility

\begin{tabular}{ccccc}
\hline $\begin{array}{c}\text { S. } \\
\text { No }\end{array}$ & $\begin{array}{c}\text { Cluster Head Changes } \\
\text { No of times } \\
\text { cluster Head } \\
\text { changes }\end{array}$ & $\begin{array}{c}\text { Pause } \\
\text { time } \\
(\mathrm{sec})\end{array}$ & $\begin{array}{c}\text { No of times } \\
\text { cluster } \\
\text { changes }\end{array}$ & $\begin{array}{c}\text { Pause } \\
\text { time } \\
(\mathrm{sec})\end{array}$ \\
\hline 1 & 15 times & 0 & 40 times & 0 \\
2 & 15 times & 20 & 30 times & 20 \\
3 & 7 times & 40 & 17 times & 40 \\
4 & 5 times & 60 & 16 times & 60 \\
5 & 3 times & 80 & 10 times & 80 \\
6 & 0 times & 100 & 0 times & 100 \\
\hline
\end{tabular}

\subsection{Queuing among clusters}

In the proposed MRED scheme, there are several parameters which will affect the performance. The queuing is done in between the gateway nodes of different clusters which will get updated by the cluster head as shown in previous section. In this section, we will try to determine their optimal values. Moreover, our scheme for estimating the average queue size of the neighborhood queue is realized by estimating the channel utilization. Major parameters are TIME INTERVAL and QUEUE SIZE as shown in Figure 5. As the main goal of this scheme is to achieve low average delay and high throughput, in order to which MRED gateways measures $\mathrm{s}_{\mathrm{ag}}$, and drops/marks the arriving

Packets with the probability $p$ to notify TCP end of the initial congestion when $s_{a g}>m_{a n}$.

We have to calculate the $\mathrm{q}_{\text {avg }}$ to find out the number of packet (in queue) transferred per unit time.

$$
\mathrm{q}_{\text {avg }}=(1-\mathrm{w}) \mathrm{q}^{\prime}{ }_{\mathrm{avg}}+\mathrm{w}_{\mathrm{q}}
$$

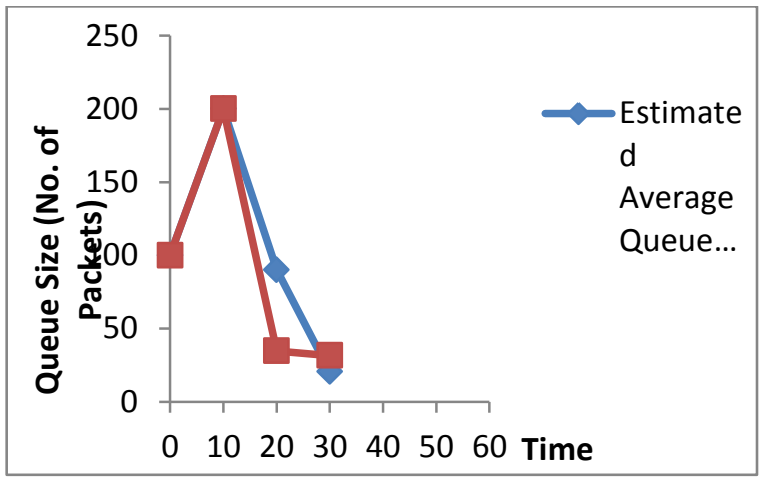

Figure 5. Queue size vs time

Table 3. Queue size Vs Time

\begin{tabular}{lrrrr}
\hline $\begin{array}{l}\text { Time } \\
\text { Estimated Average }\end{array}$ & 0 & 10 & 20 & 30 \\
$\begin{array}{l}\text { Queue Size } \\
\text { Real Average } \\
\text { Queue Size }\end{array}$ & 100 & 200 & 90 & 20 \\
\hline
\end{tabular}

In graph of Figure 4 of data represented in Table 3 increased mobility low size in clusters is seen and node changes accordingly, Queu size decseases as per pause time decrease. Thus, from Fig. 5 it's clear that initially the mobility is there packet size increase with time passes and increases Queue size decreases drop probability also increases.

\section{RESULTS ANDTHROUGHPUT ANALYSIS}

After MRED is applied, we observe that the fairness indices under the both scenarios are improved quickly along with the increase of $\mathrm{p}_{\max }$. For the hidden terminal scenario, the fairness index is close to 1 (the highest value) after $\mathrm{p}_{\max }$ is larger than 0.1 . For the exposed terminal scenario, fairness index is also above 0.95 when $\mathrm{p}_{\max }$ is larger than 0.14 . The throughput loss comes from two reasons. First, before a packet is

Analysing Mobile Random Early Detection for Congestion Control in Mobile Ad-hoc ... (Saurabh Sharma) 
dropped by NRED, it may have used the channel. Dropping such packets certainly wastes some bandwidth. Second, the NRED scheme tends to keep the wireless channel slightly underutilized. Thus, a small fraction of bandwidth is also sacrificed. (MRED) and (with NRED) show the dynamics of the two connections by plotting the instantaneous throughput of each flow as it has been shown in [21]. From Figure 6, we observe that when node 5 moves down, the two connections are out of interference with each other.

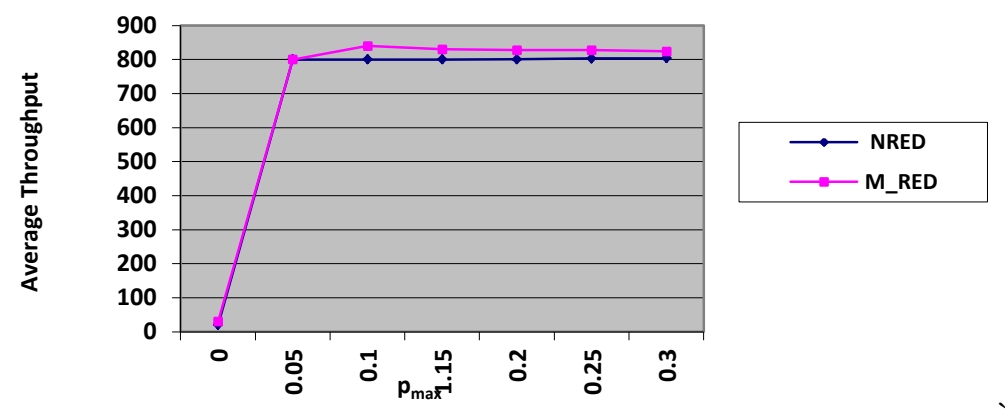

Figure 6. Average throughput vs $\mathrm{p}_{\max }$

Table 4. Average Throughput Vs $\mathrm{p}_{\max }$

\begin{tabular}{lrrrrrrr}
\hline Pmax & 0 & 0.05 & 0.1 & 1.15 & 0.2 & 0.25 & 0.3 \\
\hline NRED & 20.4 & 800 & 800 & 800 & 801 & 803 & 804 \\
MRED & 30.6 & 800 & 840 & 830 & 828 & 828 & 824 \\
\hline
\end{tabular}

The throughput of the proposed MRED is checked in comparision to NRED and it has been found that the performance of the MRED is improved 3\%. As in case, of MRED the clustering and queuing make the results so improved. In certain topology several bottleneck neighbourhoods may be present at the same time. The overall throughput of each flow is given in following Figure 7.

\section{CONCLUSION}

This analysis concludes that the overall results of the average throughput is increased using MRED and the results are compared with the previously evolved NRED. The performance of the network in terms of Queue size decreased as compared to previous results. This shows that the mobility and ad hoc nature helps in strengthening the congestion but, the clustering and queuing when applied simultaneously diminishes the effect of mobility as well as ad hoc nature as shown by the results. etc. Especially, this work may be extended for the clustered environment solution. Furthermore, the effect of multiple Gateways is also need to be considered as an important aspect to be considered. As there can be multiple gateways available with Internet access in a MANET, Handover is necessary and its effect must be considered also. Thus, it may be said that the congestion may be somehow affected or controlled in the proposed approach.
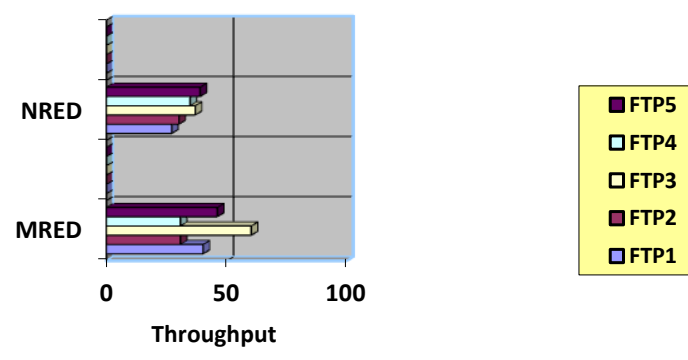

Figure 7. Performance measurement using MRED and NRED 


\section{ACKNOWLEDGEMENTS}

This work could not be done with out the day and night efforts of all authors, specially Dr. Rashi agarwal had put her long research experience to make this paper novel, Mr. Saurabh Sharma, gave a central idea of the same. Second author of this paper had contributed in real time data sampling and collection; generate the real data on NS2 continuous simulations.

\section{REFERENCES}

[1] R. N. Devikar, et al. "Issues in Routing Mechanism for Packets Forwarding: A Survey", International Journal of Electrical and Computer Engineering (IJECE), 2016, vol. 6, pp. 421-430.

[2] C. Cetinkaya, "Multi-channel cooperative MAC protocol for wireless LANs", Ad Hoc Networks 2015, vol. 28, pp. 17-37.

[3] S. Sharma, Dr. R. Agarwal, "Analysing Qos Parameters in MANETS: A Survey”, Second International Conference on (RTSTMSD-15), Recent Trends in Science, Technology, Management \& Social Development 2015, vol. 1, no. 1 , pp. 82-89.

[4] P. K. Suri, Dr. M. K.Soni, and Parul Tomar, "Cluster Based QoS Routing Protocol for MANET", International Journal of Computer Theory and Engineering, October, 2010; 2: 5.

[5] A. A. Hanbali, E. Altman, and P. Nain, "A Survey of TCP over Ad Hoc Networks", IEEE Communications Surveys and Tutorials 2005, vol. 7, no. 3, pp. 22-36.

[6] K. Xu, M. Gerla, L. Qi, and Y. Shu, "Enhancing TCP fairness in ad hoc wireless networks using neighborhood RED”, Proc. ACMMobiCom, 2003, vol. 1, no. 1, pp. 16-28.

[7] Venkata N. Padamanabhan and Randy H. Katz, "TCP Fast Start: A Technique for Speeding up Web Transfers", Proc. of IEEE Globecom'98 Internet Mini-Conference 1998.

[8] M. Chatterjee, S.K. Das and D. Turgut, "An on-demand weighted clustering algorithm (WCA) for ad hoc networks", in Proc. of IEEE GLOBECOM 2000, San Francisco November 2000, vol. 1, pp. 1697-1701.

[9] W. Feng, D. Kandlur, D. Saha, and K. G. Shin, "A self-configuring RED gateway", in Proceedings of the IEEE Conference on Computer Communications (INFOCOM '99) 1999, vol. 3, pp. 1320-1328.

[10] S. Floyd, R. Gummadi, and S. Schenker, “Adaptive RED: an algorithm for increasing the robustness of RED's active queue management," Technical Report, 2001, vol. 5, pp. 7-17.

[11] J. Chen, C. Hu, and Z. Ji, "An improved ARED algorithm for congestion control of network transmission," Mathematical Problems in Engineering 2010 (20), Article ID 329035, pp. 14-28.

[12] Christos Antonopoulo and Stavros Koubias, "Congestion Control Framework for Ad-Hoc Wireless Networks," Wireless Personal Communication 2010, vol. 52, pp. 753-775.

[13] M. Chatterjee, S. Das, and D. Turgut, "WCA: a weighted clustering algorithm for mobile Ad-hoc networks," Journal of Cluster Computing (Special Issue on Mobile Ad hoc Networks), 2002, vol. 5, pp. 193-204.

[14] A. Parekh, "Selecting routers in ad hoc wireless networks", In Proc. of the SBT/IEEE Internationalm Telecommunications Symposium, 1994.

[15] A. Ephremides, J.E. Wieselthier, D.J. Baker. "A design concept for reliable mobile radio networks with frequency hopping signalling," In Proceedings of the IEEE 1987, vol. 75, pp. 56-73.

[16] Liang Guo and Ibrahim Matta, "The War Between Mice and Elephants," in Proc. of the Ninth IEEE International Conference on Network Protocols ICNP, 2001, 1, pp. 180-188.

[17] A. M. Borah, Bobby Sharma \& Manab Mohan Borah, "A congestion Algorithm for Moility Model in Mobile Ad hoc Networks", International Journal of Computer Application, vol. 118, no. 23, May 2015, pp. 27-31.

[18] S. Subburam, P.S. A. Khader, "Predictive congestion Control mechanism for MANETS", IJCSE, vol. 3, no. 5, pp. 640-648.

[19] A. Ramil, N.Zulkifli, S. M. Idrus, "Power Comsumption Modeling and analysis of Integrated Optical- Wireless Access Network", IJECE, December 2017, vol. 7, no. 6, pp. 3475-3483.

[20] Roshni Agarwal, Pratuysh Sharma, Vijay Malviya, "A Novel Method for Queue Management Using RED Technique In Mobile Ad Hoc Network”, I-SMAC(IoT in Social, Mobile, Analytics \& Cloud), IEEE, 2017, 17, pp. 175-180.

[21] R. Rana, et al., "Analyzing AODV in the presence of Malicious node in MANETS", Second International Conference on (RTSTMSD-15), Recent Trends in Science, Technology, Management \& Social Development, 2015, 1 , pp. 82-89.

\section{BIOGRAPHIES OF AUTHORS}

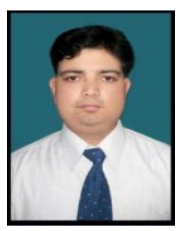

Mr. Saurabh Sharma is the research scholar in the comouter science department of Sharda University. Done M. tech in Information technology. He has done MCA from MDU, Rohtak, and done Graduate degree in Mathematics also, done CISCO (CCNA \& CCAI).) certification and its academic training from CISCO Regional Academy, Amity University. Authored 3 Books on Information Security and 15 research papers . A number of other certifications are there under his name. His Favourate fields of interest are Networking, Ad hoc networks, Wireless Networking, Information Security \& cryptography etc. 


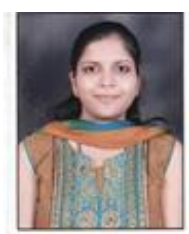

Dipti Gupta received the B.Tech degree in Information Technology from Uttar Pradesh Technical University, Lucknow, India, in 2007. She also received the M.Tech degree in Computer Science \& Engineering from Sharda University, Greater Noida, India, in 2012. Currently, she is an assistant professor in Computer Science \& Engineering Department at Skyline Institute of Engineering \& Technology, Greater Noida, India. Her research interests include wireless networks, mobile ad hoc networks and cryptography.

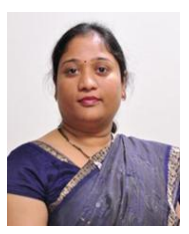

Rashi Agarwal Ph. D. computer Science from Gautam Budh Technical University, Lucknow. She is an eminet scholar of computer applications and Done her PG from renound govt. Instution of state of Uttar Pradesh. She has written a number of research papers and books in the field of computer applications and information technology. Her research interests include Software Engineering, , Programming networks and Cryptography.

\section{Appendix}

Table 1. Related Literature Review

\begin{tabular}{|c|c|c|c|c|}
\hline S. No & Author \& Year & Research Topic & Methodology & Limitations \\
\hline 1 & $\begin{array}{l}\text { Venkata N. Padamanabhan, } \\
\text {, Randy H. Katz,1998 }\end{array}$ & $\begin{array}{l}\text { TCP Fast Start: A Technique for } \\
\text { Speeding Up Web Transfers }\end{array}$ & TCP fast start & $\begin{array}{l}\text { risk of performance } \\
\text { degradation }\end{array}$ \\
\hline 2 & $\begin{array}{l}\text { S. Floyd and V. Jacobson, } \\
1993\end{array}$ & $\begin{array}{l}\text { Random Early Detection Gateways } \\
\text { for Congestion Avoidance }\end{array}$ & RED Queuing & $\begin{array}{l}\text { No bound of threshold } \\
\text { value }\end{array}$ \\
\hline 3 & $\begin{array}{l}\text { W. Feng, D. Kandlur, D. } \\
\text { Saha, and K. G. Shin, } 1999\end{array}$ & A self-configuring RED gateway & ARED Queuing & For wired network \\
\hline 4 & $\begin{array}{l}\text { S. Floyd, R. Gummadi, and } \\
\text { S. Schenker, } 2001\end{array}$ & $\begin{array}{l}\text { Adaptive RED: an algorithm for } \\
\text { increasing the robustness of RED's } \\
\text { active queue management }\end{array}$ & $\begin{array}{l}\text { Revised ARED using } \\
\text { AIMD mechanism }\end{array}$ & For wired network \\
\hline 5 & $\begin{array}{l}\text { J. Chen, C. Hu, and Z. Ji, } \\
2010\end{array}$ & $\begin{array}{l}\text { An improved ARED algorithm for } \\
\text { congestion control of network } \\
\text { transmission }\end{array}$ & $\begin{array}{l}\text { Improved ARED } \\
\text { Queuing }\end{array}$ & For wired network \\
\hline 6 & $\begin{array}{l}\text { Liang Guo and Ibrahim } \\
\text { Matta, } 2001\end{array}$ & The War Between Mice and Elephants & Weighted RED Queuing & For wired network \\
\hline 7 & $\begin{array}{l}\text { Christos Antonopoulo and } \\
\text { Stavros Koubias, } 2010\end{array}$ & $\begin{array}{l}\text { Congestion Control Framework for } \\
\text { Ad-Hoc Wireless Networks }\end{array}$ & $\begin{array}{l}\text { Cross layer Design } \\
\text { approach }\end{array}$ & $\begin{array}{l}\text { Only Static nodes are } \\
\text { covered }\end{array}$ \\
\hline 8 & $\begin{array}{l}\text { K. Xu, M. Gerla, L. Qi, and } \\
\text { Y. Shu, } 2003\end{array}$ & $\begin{array}{l}\text { Enhancing TCP fairness in ad hoc } \\
\text { wireless networks using neighborhood } \\
\text { RED }\end{array}$ & NRED Queuing & static nodes \\
\hline 9 & $\begin{array}{l}\text { Prof. P.K. Suri, Dr. M. } \\
\text { K.Soni, and Parul Tomar, } \\
2010\end{array}$ & $\begin{array}{l}\text { Cluster Based QoS Routing Protocol } \\
\text { for MANET }\end{array}$ & CBQR & QoS is tested \\
\hline
\end{tabular}

